Diabetes and OSA

\section{Diabetes and sleep apnoea: a hidden epidemic?}

\section{J Wilding}

\section{A diagnosis of OSA should be considered in patients with type 2 diabetes}

t

is now well established that obstructive sleep apnoea (OSA) is associated with an increased risk for cardiovascular disease, and that this is probably related to the fact that patients with sleep apnoea are likely to have a high prevalence of the risk factors that comprise the metabolic syndromenamely, central adiposity, dyslipidaemia (low HDL cholesterol, raised triglycerides), high blood pressure, insulin resistance, and hyperglycaemia-even after correction for adiposity. ${ }^{1-3}$ Type 2 diabetes is also typically associated with a similar metabolic profile, so it is not surprising that the prevalence of diabetes in obese hypertensive patients with sleep apnoea may be as high as $40 \%{ }^{4}$ Given that both type 2 diabetes and OSA are highly prevalent in the general population, it is important to establish the prevalence of OSA in people with type 2 diabetes as this may represent a significant population with undiagnosed sleep apnoea that may be amenable to treatment.

In this issue of Thorax West and colleagues $^{5}$ have attempted to establish the prevalence of OSA in a community and hospital based population of men with established type 2 diabetes. Initial screening was by questionnaire to over 1600 men with diabetes, which achieved an overall response rate of $56 \%$; this identified populations of "high risk" and "low risk" subjects based on selfreported height, weight, and sleep related symptoms. A subset of each group was invited for a formal sleep study. OSA was found in $31 \%$ of high risk patients and $13 \%$ of low risk patients; the overall prevalence of OSA in the diabetes population was estimated at $23 \%$ compared with $6 \%$ in a community based prevalence study conducted a few years earlier.

This study does have limitations related to possible selection bias-for example, the prevalence of OSA in nonresponders to the questionnaire is unknown, and most of the patients with diabetes were cared for in secondary care and may have had more severe disease than would be expected in a purely community based sample. Furthermore, the use of historical controls at a time when the prevalence of obesity in the population was rising rapidly may have underestimated the current prevalence of OSA in the nonseems likely that the overall conclusions of this study are valid, and that OSA is highly prevalent in men with type 2 diabetes.

As expected, OSA was more common in the "high risk" group who had more symptoms and were more likely to be obese, but there was only a weak correlation with glycaemic control as assessed by HbAlc, which is perhaps unsurprising given the confounding effects of oral hypoglycaemic drugs and insulin that are used to treat diabetes. It is disappointing that the questionnaires used to identify high and low risk groups for likelihood of OSA were relatively insensitive; however, the high risk group had a greater proportion of patients who reported sleepiness, and this is the group who would be offered treatment under current guidelines. ${ }^{6}$ West and colleagues ${ }^{5}$ did not include women with diabetes in their study and, although sleep apnoea is generally less common in women than in men, it is important that future research also addresses this issue in women.

The results of this study have important implications for those treating people with type 2 diabetes in both primary and secondary care. Clinicians should consider the possible diagnosis of OSA in patients with type 2 diabetes and, in patients who are symptomatic, determine whether treatment should be offered. This may not just improve sleep related symptoms but also has the potential to improve some features of the metabolic syndrome, notably blood pressure and possibly insulin resistance. $^{7-9}$

The mechanisms that underlie the association between type 2 diabetes and OSA are likely to be complex; both develop in the context of the "common soil" of obesity, but there is evidence to support the hypothesis that sleep diabetic population. Nevertheless, it apnoea may predispose to diabetes, and that diabetes increases the risk of sleep apnoea. There is a growing body of research that supports the concept that reduced sleep duration may itself increase the risk of diabetes, possibly via hormonal mechanisms related to stress such as catecholamines and cortisol. ${ }^{10}$ In addition, arousals in the context of sleep apnoea will activate the sympathetic nervous system and this could contribute both to insulin resistance and sympathetic mediated impairment of $\beta$ cell function. ${ }^{11}$ Both insulin resistance and sleep apnoea have been associated with increased circulating concentrations of pro-inflammatory cytokines, ${ }^{12}$ some of which may derive from adipose tissue and may be secreted in greater amounts in response to tissue hypoxia. ${ }^{13}$ Adiponectin, a recently discovered adipose tissue derived hormone, has been shown to protect against the development of insulin resistance; one small study has shown lower adiponectin concentrations in patients with OSA than in those with simple snoring, implying that low levels of this hormone might contribute to the development of insulin resistance in OSA. ${ }^{14}$ Moreover, adiponectin secretion may also be reduced by hypoxia. ${ }^{15}$ Physical activity is strongly protective against the development of insulin resistance and type 2 diabetes, but the possibility that individuals with OSA are less physically active as a result of daytime sleepiness has not been explored. Diabetes can result in autonomic dysfunction, and this has been shown to result in abnormalities in respiratory function, particularly in the response to hypoxia and hypercapnia that may have implications for the development of symptomatic OSA which appears to be common even in non-obese diabetic patients with autonomic neuropathy. ${ }^{1617}$

There seems little doubt that patients with diabetes are more likely to have sleep apnoea than the general population. This has important implications for public health, especially given the prevalence rates of OSA reported by West et al of 23\% in the diabetes population. In the UK alone, with an estimated 3 million people with type 2 diabetes, this represents nearly three quarters of a million individuals. Preliminary evidence suggests that treatment of OSA in people with type 2 diabetes improves glycaemic control, as well as reducing blood pressure and improving sleep related symptoms. ${ }^{18}$ In a largely non-diabetic population with severe OSA, long term use (over 10 years) of continuous positive airways pressure (CPAP) ventilation was associated with a more than $50 \%$ lower incidence of both fatal and non-fatal cardiovascular disease. ${ }^{19}$ Given that cardiovascular disease 


\section{EDITORIAL}

is the main cause of death in people with type 2 diabetes, it is possible that the beneficial effects of CPAP treatment in diabetic patients with OSA extend beyond improving symptoms relating to sleepiness. There is therefore a clear need to undertake further prevalence studies in an unselected group of patients with type 2 diabetes and then to investigate which subjects will benefit from treatment, both from a clinical and from a health economic perspective, using carefully designed studies with robust methodology and long term follow up.

Thorax 2006;61:928-929.

doi: 10.1136/thx.2006.062513

Correspondence to: Professor J Wilding, School of Clinical Sciences, Clinical Sciences Centre University Hospital Aintree, Liverpool L9 7AL, UK; i.p.h.wilding@liv.ac.uk

Competing interests: none.

\section{REFERENCES}

1 Newman AB, Nieto FJ, Guidry U, et al. Relation of sleep-disordered breathing to cardiovascular disease risk factors: The Sleep Heart Health Study. Am J Epidemiol 2001;154:50-9.
2 Coughlin SR, Mawdsley L, Mugarza JA, et al. Obstructive sleep apnoea is independently associated with an increased prevalence of metabolic syndrome. Eur Heart J 2004;25:735-41.

3 Punjabi NM, Sorkin JD, Katzel LI, et al. Sleepdisordered breathing and insulin resistance in middle-aged and overweight men. Am J Respir Crit Care Med 2002; 165:677-82.

4 Elmasry A, Lindberg E, Berne C, et al. Sleep disordered breathing and glucose metabolism in hypertensive men: a population-based study. J Intern Med 2001;249:153-61.

5 West SD, Nicoll DJ, Stradling JR. Prevelance of obstructive sleep apnoea in men with type 2 diabetes. Thorax 2006;61:945-50.

6 Scottish Intercollegiate Guidelines Network (SIGN). Management of obstructive sleep apnoea/hypopneoa syndrome in adults: a national clinical guideline, 2003. http:// www.sign.ac.uk/pdf/sign73.pdf.

7 Ip MSM, Lam B, Ng MMT, et al. Obstructive sleep apnea is independently associated with insulin resistance. Am J Respir Crit Care Med 2002; 165:670-6.

8 Harsch IA, Schahin SP, Bruckner K, et al. The effect of continuous positive airway pressure treatment on insulin sensitivity in patients with obstructive sleep apnoea syndrome and type 2 diabetes. Respiration 2004;71:252-9.

9 Pepperell JC, Ramdassingh-Dow S, Crosthwaite N et al. Ambulatory blood pressure after therapeutic and subtherapeutic nasal continuous positive airway pressure for obstructive sleep apnoea: a randomised parallel trial. Lancet 2002;359:204-10

10 Yaggi HK, Araujo AB, McKinlay JB. Sleep duration as a risk factor for the development of type 2 diabetes. Diabetes Care 2006;29:657-61.
11 Lopez-Jimenez F, Somers VK. Stress measures linking sleep apnea, hypertension and diabetes: AHI vs arousals vs hypoxemia. Sleep 2006;29:743-4.

12 Boudjeltia KZ, Van Meerhaeghe A, Doumit S, et al. Sleep apnoea-hypopnoea index is an independent predictor of high-sensitivity C-reactive protein elevation. Respiration 2006;73:243-6.

13 Trayhurn P, Wood IS. Signalling role of adipose tissue: adipokines and inflammation in obesity. Biochem Soc Trans 2005;33:1078-81.

14 Zhang XL, Yin KS, Wang $H$, et al. Serum adiponectin levels in adult male patients with obstructive sleep apnea hypopnea syndrome. Respiration 2006;73:73-7.

15 Chen BY, Lam KSL, Wang Y, et al. Hypoxia dysregulates the production of adiponectin and plasminogen activator inhibitor-1 independent of reactive oxygen species in adipocytes. Biochem Biophys Res Commun 2006;341:549-56.

16 Bottini P, Dottorini ML, Cordoni MC, et al. Sleepdisordered breathing in nonobese diabetic subjects with autonomic neuropathy. Eur Respir J 2003;22:654-60

17 Weisbrod CJ, Eastwood PR, O'Driscoll G, et al Abnormal ventilatory responses to hypoxia in type 2 diabetes. Diabet Med 2005;22:563-8.

18 Babu AR, Herdegen J, Fogelfeld L, et al. Type 2 diabetes, glycemic control, and continuous positive airway pressure in obstructive sleep apnea. Arch Intern Med 2005; 165:447-52.

19 Marin JM, Carrizo SJ, Vicente E, et al. Long-term cardiovascular outcomes in men with obstructive sleep apnoea-hypopnoea with or without treatment with continuous positive airway pressure: an observational study. Lancet 2005;365: 1046-53. 